\title{
Knowledge, Prevention Practice and Associated Factors of Stroke Among Hypertensive and Diabetic Patients - A Systematic Review
}

\author{
Abreham Degu Melak (D) \\ Dawit Wondimsigegn (iD ${ }^{2}$ \\ Zemene Demelash Kifle' \\ 'University of Gondar, College of \\ Medicine and Health Science, School of \\ Pharmacy, Department of Pharmacology, \\ Gondar, Ethiopia; ${ }^{2}$ University of Gondar, \\ College of Medicine and Health Science, \\ School of Pharmacy, Department of \\ Pharmaceutics and Social Pharmacy, \\ Gondar, Ethiopia
}

\begin{abstract}
Introduction: Because of the inadequate level of public awareness of the disease, the incidence of stroke has been sharply rising. Eventually, due to the prehospital delay, many stroke cases could not be eligible for thrombolysis thereby poor rehabilitative outcome has been tremendously increased. Thus, this study aimed to review the level of knowledge, prevention practice, and associated factors of stroke among hypertensive and diabetic patients.
\end{abstract}

Methods: A systematic review of primarily published articles (2010-2020) related to knowledge and prevention practices of stroke was performed by searching online electronic databases like PubMed, Google Scholar, Refseek, Science direct, ResearchGate, and manual Google search by using the keywords and MeSH terms. Studies conducted on knowledge and prevention practices amongst hypertensive and/or diabetic patients were included.

Results: Out of 531 searched studies, 42 articles were identified to be reviewed. The reported overall knowledge of stroke was ranging from $4.4 \%$ to $79 \%$. Knowledge to the signs/symptoms of stroke was $23.6 \%$ to $87 \%$. However, $15 \%$ to $77 \%$ of subjects were also reported that they did not know any sign of stroke. The range of risk factor knowledge was $10.5 \%$ to $86.6 \%$. The reported level of stroke prevention practice was $2.4 \%$ to $72 \%$ but physical activity and weight reduction practice were relatively low. Inadequate level of knowledge and prevention practice of stroke was related to elderly, female gender, uneducated, unmarried, rural residents, economically low, comorbidity and unemployed individuals.

Conclusion: The current finding revealed that the level of knowledge and prevention practice of stroke was inadequate. Hence, the finding highlights health educational programs should be planned as an important avenue to enhance stroke awareness among the high-risk populations.

Keywords: stroke, knowledge, warning sign, risk factor, prevention

\section{Introduction}

Despite global age-standardized mortality rates of stroke had shown a sharp decline from 1990 to 2016, its prevalence rate and burden have been still high. ${ }^{1}$ Reports showed that $80 \cdot 1$ million prevalent cases, 5.5 million deaths, and $116 \cdot 4$ million DALYs of stroke in the world were recorded at the end of 2016. Its burden in developing countries especially Sub-Saharan Africans (SSA) is still high. ${ }^{2,3}$

Although stroke is considered as multi-factorial, it is majorly attributed to the contributing risk factors like uncontrolled hypertension (HTN), diabetes mellitus
Correspondence: Abreham Degu Melak University of Gondar, College of Medicine and Health Science, School of Pharmacy, Department of Pharmacology, Gondar, Ethiopia

Email abrishdegu8@gmail.com 
(DM), dyslipidemia, heart disease, and other modifiable risk factors. The highest contributor is HTN which accounts for up to $75 \%$ of stroke cases. ${ }^{4-8}$ Moreover DM contributes up to $30 \%$ incidence to stroke events. ${ }^{1,9-13}$

Therefore, prior awareness in these high-risk populations can have a very important implication to decrease the incidence of stroke emergencies. Creating awareness about stroke helps to promote early hospital presentation thereby reduces long-term complications of the disease. Due to the inability to know the disease condition 50$87 \%$ of patients presented to the hospital out of the window period of recombinant Tissue Plasminogen Activator (tPA). As a result, they could not be eligible to the American Heart Association and American Stroke Association (AHA/ASA) recommended treatment protocol. $^{14-16}$

Poor recognition of warning signs of stroke often results in a delay in hospital presentation so that increases strokerelated morbidity and mortality. Extensive studies devoted to assessing the knowledge status of patients admitted on stroke and were reported as they had not had information on stroke disease and the way how to prevent it. ${ }^{17-20}$ More than $70 \%$ of stroke patients did not know risk factors and 20-25\% were smokers and alcoholics. $^{21}$

Increasing awareness towards risk factors, warning signs, and prevention strategies of the stroke to the highrisk populations can imply a reduction of its incidence and pre-hospital care delay. ${ }^{22-25}$ Therefore, this study aimed to review the level of knowledge, prevention practice, and associated factors towards stroke among hypertensive and diabetic populations.

\section{Method}

This systematic review was reported with the recommended protocols of Preferred Reporting Items for Systematic Reviews and Meta-Analyses (PRISMA). ${ }^{26}$

\section{Information Source and Search Strategy}

A search strategy was developed to look at recent existing articles and adapted to search, PubMed, Google Scholar, ResearchGate, Refseek, Science direct, and Manual google search by using keywords, indexing terms (MeSH terms), and the combination of them. Keywords were, "Stroke", "Knowledge", "Awareness", "Risk Factor", "Warning Sign", "Primary Prevention", "Lifestyle", "Self-Care", "Hypertension", "Diabetic Mellitus". An advanced searching method by Boolean operators (AND \& OR) was also used; "Stroke AND Awareness", Stroke AND
Knowledge", "Stroke AND Awareness AND Hypertension OR Diabetic", "Stroke AND Prevention". Two reviewers independently reviewed the titles and abstracts of the articles identified by these searching databases. Finally, any article that appeared to meet the inclusion criteria was read in detail.

\section{Inclusion and Exclusion Criteria}

Primary studies published between 2010 and 2020 were included. The assessment questions used to test knowledge and practice could be open-ended, closed, or multiple choice. The way of delivering the questions could be through telephone, face-to-face, or postal surveys. We excluded articles that could not be accessed full text because of the limited data that could be extracted. Studies published other than English could be excluded due to the poor ability of reviewers in other languages. Non-empiric citations including editorials, letters, commentaries, and opinions were also excluded.

\section{Study Selection and Quality Assessment}

We used Endnote version 7 reference manager to remove duplicated articles as shown in Figure 1. The authors assessed the quality of each study independently. If any discrepancy happened, it was resolved through consensus discussions. The methodological quality of all the reviewed studies was checked by the National Institute of Health (NIH) Quality Assessment Tool for Observational Cohort and Cross-Sectional Studies before inclusion in the review. ${ }^{27}$ The tool has 14 questions to be rated as Yes, No, Not applicable, and Not reported. Of course, it does not have a numerical scale, the general guidance provides to determine the overall quality of studies and to be grade as good, fair, and poor. Accordingly, studies answer more questions positively graded as Good $(\geq 7)$ followed by Fair $(5-6)$ and poor quality $(\leq 4)$ as it was adapted from a previous study. ${ }^{28}$

\section{Data Extraction and Synthesis}

The authors looked at and screened the articles independently based on the inclusion/exclusion criteria. The following details were extracted from each study using an abstraction format prepared in Microsoft excel: author, year of publication, study area, study subjects, sample size, study design, sampling technique, general and/or overall knowledge of stroke, knowledge towards risk factors, knowledge towards the signs and symptoms of stroke, knowledge towards stroke prevention, lifestyle 
practices of stroke prevention and associated factors related to knowledge and prevention practices.

After the raw data collected from the included articles, the results were synthesized into tables as per the objectives of the review. Finally, the studies included in the review were categorized under one or more of the following topics to be narratively synthesized; knowledge of general concepts of stroke, knowledge of risk factors of stroke, knowledge of stroke sign/symptoms, knowledge of the pre-stroke prevention, and lifestyle modification practice (LMP) of stroke prevention. It was impossible to express the result quantitatively in the form of metaanalysis since the reported data was insufficient.

\section{Result}

A total of 531 titles were identified in the searching databases. After duplicates and irrelevant articles were screened, 42 eligible studies were left to be reviewed for knowledge and prevention practice as shown in Figure 1.

\section{Risk of Bias}

High risk of bias can be expected in studies where the quality assessment element was not reported as per the standards. In the contrary studies that were rated as yes (reported) in the quality assessment questions considered as low risk of bias. As a result, more than $80 \%$ of the studies had clearly settled objectives, ${ }^{17,29-69}$ specified

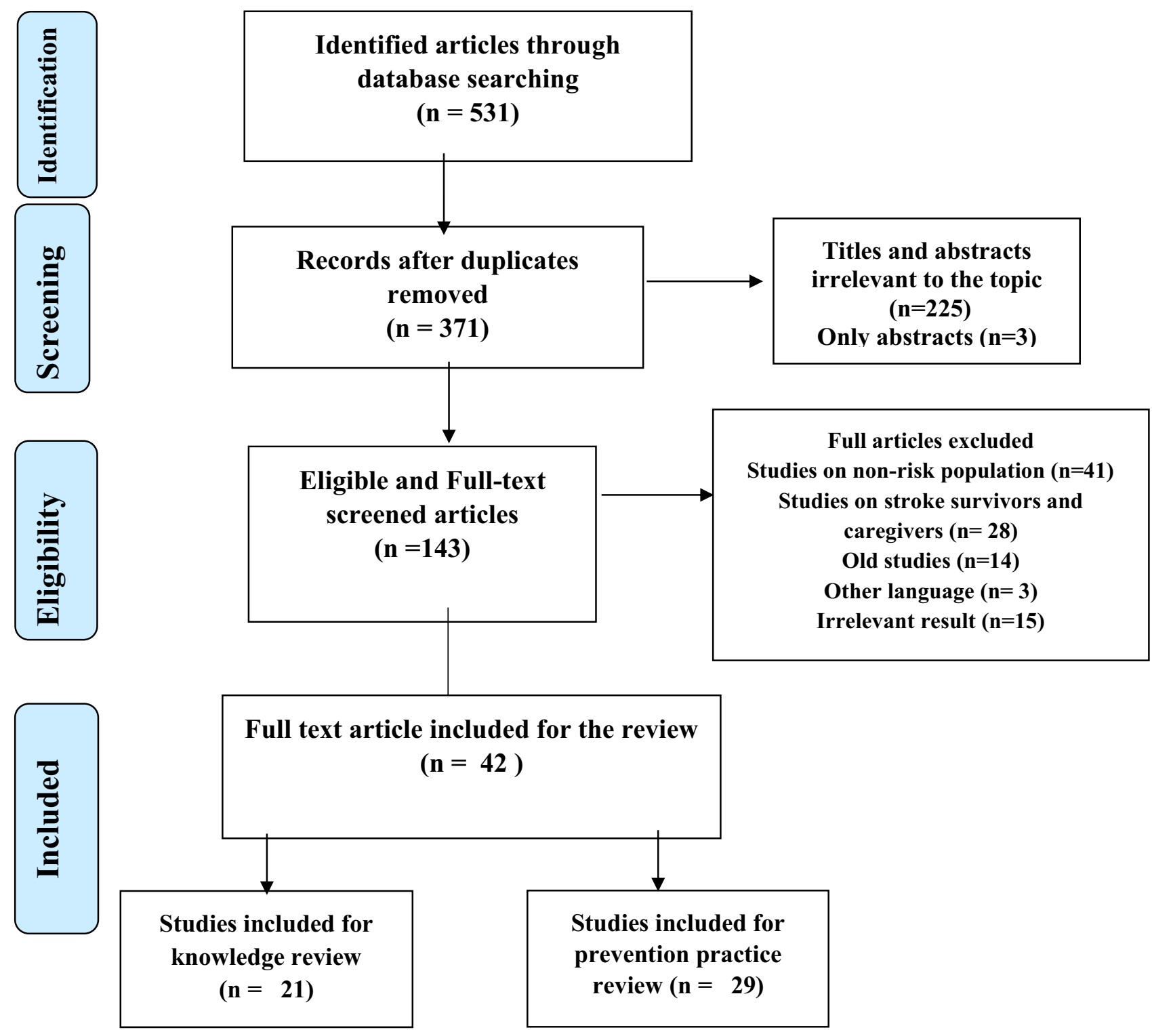

Figure I Flow chart of the article selection process. 
study populations, ${ }^{17,29-35,37,39-53,55,56,58,60-64,66-69}$ inclusion/exclusion criteria, ${ }^{17,29-35,37,40,42-51,53-57,59,61-69}$ adjusted confounders, ${ }^{17,29-69}$ defined dependent variables, ${ }^{17,29,30,32-44,46-51,53,55-58,63-69}$ and independent variables. ${ }^{17,29-31,33,34,36,37,39-44,46-49,51-60,63-65,69}$ Whereas $\geq 50 \%$ participation rate, ${ }^{29-31,33-35,37,39-42,44,46,48,50-}$ $53,55,56,61-63,65,68,69$ justified sample size determination, ${ }^{29-}$ $32,34,37-40,42-44,46-52,55-57,60,61,63,65,66,68,69$ and less $20 \%$ lost follow up/response rate, ${ }^{17,29-31,34-36,39,47,49-51,56,66,68}$ were clearly stated in $61.9 \%, 69 \%$ and $35.7 \%$ of studies, respectively. Other questions were not applicable for our reviewed studies. Finally, after critically appraised near to $62 \%, 31 \%$ and $7 \%$ of studies were found in good, fair, and poor quality, respectively.

\section{Characteristics of Studies}

The reviewed 42 articles comprised a total of 10,063 sampled participants obtained from 20 different world countries; 20 Asian, 16 African, 3 European, 2 North American, and 1 South American study. Twenty-two studies (52.4\%) were done among hypertensive patients, ten studies $(23.8 \%)$ among diabetic patients, and ten $(23.8 \%)$ of the studies were done among hypertensive and diabetic patients. The mean age of participants was $55.1 \pm 5.7$ years per study. The sex proportion of the subjects was $53 \pm 14.5 \%$ of males and $49.1 \pm 12 \%$ of females. The search results revealed that most of these articles were institutionally based and were designed as a cross-sectional study. However, their randomization technique was a probabilistic and non-probabilistic method as mentioned in Table 1.

\section{Overall Knowledge Towards Stroke}

Overall knowledge can be operationalized as knowing pick general concepts obtained from inquiries regarding the definition, cause, organs affected, and preventions of stroke. Of the reviewed studies, seven studies reported the calculated overall good knowledge of respondents towards stroke. As good awareness was shown in Ghana $(79.18 \%),{ }^{33}$ low awareness in Iraq $\left.(4.4 \%)\right)^{32}$ Nigeria $(16.9 \%){ }^{53}$ and Ethiopia $(18.3 \%)^{29}$ were reported. The participants' scope of knowledge that stroke affects the brain was also reported in six studies ranged from $21.6 \%,{ }^{29}$ to $87.4 \%{ }^{34}$ With a comparative cross-sectional study aimed to assess the knowledge difference of native and migrant diabetic patients towards stroke in Germany and Turkey, only $50 \%$ of the native and $25 \%$ migrant diabetic cases responded exactly that the brain is the affected organ with stroke. ${ }^{65}$ The participants' overall knowledge can be seen in Table 2 .

\section{Knowledge Towards Warning Signs of Stroke}

Seventeen studies have been employed to knowledge towards warning signs of a stroke. Out of these, eight articles revealed the computed prevalence of overall good level of knowledge of warning signs of stroke ranged from $23.6 \%{ }^{53}$ to $87 \% ;^{34}$ half of them were below $50 \%$. The ability to name the signs/symptoms of stroke markedly varied between the studies. The signs of stroke sudden half body paralysis/weakness, sudden trouble speaking, sudden severe headache, and sudden double vision were reported by more than $65 \%$ of the studies. From these, $70-85 \%$ of studies reported low knowledge $(<50 \%)$ of sudden double vision and severe headache as the signs of a stroke. Besides these, though few studies were reporting, there was low awareness detected to numbness, imbalance movement, memory loss, and dizziness. Unfortunately, eight studies depicted that $15-77 \%$ of the respondents did not know any sign of stroke as shown in Table 3.

\section{Knowledge Towards Risk Factors of Stroke}

Based on studies that stated on risk factor awareness, $10.5 \%^{32}$ to $86.6 \%{ }^{34}$ overall good levels of risk factor knowledge were reported. But only two studies were reporting more than $50 \%{ }^{34,43}$ Despite it was not adequate, just more than half of the studies reported whether their respective subjects had better knowledge of HTN as the risk of stroke. The majority of studies reported a low level of knowledge to hypercholesterolemia, DM, past stroke history, cigarette smoking, obesity, physical inactivity, bad diet, alcohol intake, family history, elderly, and heart disease. Studies inquired on stress as a risk of stroke and revealed up to $96 \%$ of knowledge. However, the review found $2.3-77 \%$ of the HTN and DM patients that did not know any risk factors of stroke as shown in Table 4.

\section{Knowledge Towards the Prevention of Stroke}

Even though few studies were enrolled in the knowledge of stroke prevention, a single study reported the overall calculated level of stroke prevention knowledge $(90.8 \%){ }^{34}$ Two authors reported $82-85 \%$ of subjects 
Table I Characteristics of Reviewed Studies

\begin{tabular}{|c|c|c|c|c|c|c|c|c|}
\hline Study, Year of Publication & Country & $\begin{array}{l}\text { Disease } \\
\text { Condition }\end{array}$ & $\begin{array}{l}\text { Sample } \\
\text { Size }\end{array}$ & $\begin{array}{l}\text { Mean } \\
\text { Age } \\
\text { (Years) }\end{array}$ & Male (\%) & Female (\%) & $\begin{array}{l}\text { Data } \\
\text { Collection }\end{array}$ & Method \& Sampling \\
\hline Abate et al, $2019^{29}$ & Ethiopia & HTN & 278 & 54.40 & 47.80 & 52.20 & Y & Cross sectional, SRS \\
\hline Aboutorabi Zarchi et al, $2019^{30}$ & Iran & DM & 281 & 54.04 & 29.60 & 70.40 & O, MCQ & Cross-sectional \\
\hline Al-Beladi et al, $2016^{31}$ & Saudi Arabia & $\begin{array}{l}\text { DM \& } \\
\text { HTN }\end{array}$ & 166 & - & 42.20 & 57.80 & O\&C & $\begin{array}{l}\text { Cross-sectional, Consecutive } \\
\text { Sampling }\end{array}$ \\
\hline Amen, $2016^{32}$ & Iraq & HTN & 114 & 58.40 & 58.8 & 45.70 & C & $\begin{array}{l}\text { Cross-sectional Purposive } \\
\text { sampling }\end{array}$ \\
\hline Ampiah et al, $2018^{33}$ & Ghana & $\begin{array}{l}\text { DM \& } \\
\text { HTN }\end{array}$ & 208 & 57.30 & 41.30 & 58.70 & C & Cross-sectional, Convenient S \\
\hline Arisegi et al, $2018^{34}$ & Nigeria & $\begin{array}{l}\text { DM \& } \\
\text { HTN }\end{array}$ & 239 & 48.21 & 34.3 & 65.7 & & Cross-sectional, SRS \\
\hline Chukwuocha, $2018^{38}$ & Nigeria & DM & 91 & 59 & 33 & 67 & O & Cross-sectional \\
\hline Dar et al, $2019^{39}$ & Pakistan & HTN & 384 & 51.4 & 52.90 & 47.10 & Y & Cross-sectional, consecutive S \\
\hline Ehidiamen et al, $2018^{43}$ & Nigeria & HTN & 144 & 58.50 & 53.50 & 46.50 & O & Cross-sectional, consecutive S \\
\hline Mersal et al, $2020^{50}$ & Saudi Arabia & HTN & 203 & 41.83 & 51.20 & 48.80 & C & Cross-sectional, \\
\hline Oparah, $2017^{53}$ & Nigeria & $\begin{array}{l}\text { DM \& } \\
\text { HTN }\end{array}$ & 148 & 54.30 & 45.90 & 54.10 & O & Cross-sectional, \\
\hline Poongothai et al, $2017^{54}$ & India & HTN & 50 & 48.7 & 72.00 & 28 & & Observational \\
\hline Venkatesh et al, $2016^{61}$ & India & HTN & 105 & 47.50 & 66.70 & 33.5 & C & Cross-sectional, consecutive S \\
\hline Vincent et al, $2015^{62}$ & Nigeria & $\begin{array}{l}\text { DM \& } \\
\text { HTN }\end{array}$ & 200 & 47.50 & 66.70 & 33.30 & O & Cross-sectional, consecutive S \\
\hline Wahab et al, $2015^{63}$ & Nigeria & $\begin{array}{l}\text { DM \& } \\
\text { HTN }\end{array}$ & 314 & 56.40 & 47.10 & 52.90 & & Cross-sectional, consecutive S \\
\hline Weltermann et al, $2013^{65}$ & Germany & DM & 218 & - & 50.40 & 49.50 & O & Cross-sectional \\
\hline Konduru et al, $2017^{47}$ & India & DM & 100 & - & 64.00 & 36.00 & & Prospective and observational \\
\hline Chimberengwa et al, $2019^{69}$ & Zimbabwe & HTN & 304 & 59.00 & 34.60 & 65.40 & & Cross-sectional \\
\hline Okonta et al, $2014^{52}$ & South Africa & DM & 217 & - & - & - & & Cross-sectional \\
\hline Tesema et al, $2016^{60}$ & Ethiopia & HTN & 130 & - & 57.70 & 42.30 & O\&C & Cross-sectional \\
\hline Buda et al, $2017^{37}$ & Ethiopia & HTN & 205 & 53.90 & 48.8 & 51.2 & & Cross-sectional \\
\hline Durai et al, $2015^{42}$ & India & HTN & 100 & 51 & 100 & NP & & Cross-sectional \\
\hline Mutyambizi et al, $2020^{68}$ & South Africa & DM & 396 & - & 39.24 & 60.76 & MCQ & Cross-sectional \\
\hline Rashidi et al, $2018^{57}$ & Iran & HTN & 110 & 57.97 & 47.3 & 52.7 & MCQ & Cross-sectional \\
\hline Faiz et al. $2018^{44}$ & Norway & $\begin{array}{l}\text { DM \& } \\
\text { HTN }\end{array}$ & 173 & 68 & 63.6 & 36.4 & O & Prospective cross sectional \\
\hline Sheeba et al, $2017^{59}$ & India & DM & 100 & - & 75 & 25 & & Cross-sectional, Convenient S \\
\hline Dinesh et al, $2016^{40}$ & India & DM & 400 & - & 62.25 & 38.75 & C & Cross-sectional, Random \\
\hline Bollampally et al, $2016^{66}$ & India & HTN & 160 & - & 47.5 & 52.5 & $\mathrm{O}$ & Prospective, observational \\
\hline
\end{tabular}


Table I (Continued).

\begin{tabular}{|c|c|c|c|c|c|c|c|c|}
\hline Study, Year of Publication & Country & $\begin{array}{l}\text { Disease } \\
\text { Condition }\end{array}$ & $\begin{array}{l}\text { Sample } \\
\text { Size }\end{array}$ & $\begin{array}{l}\text { Mean } \\
\text { Age } \\
\text { (Years) }\end{array}$ & Male (\%) & Female (\%) & $\begin{array}{l}\text { Data } \\
\text { Collection }\end{array}$ & Method \& Sampling \\
\hline Warren-Findlow et al, $2011^{64}$ & Carolina & HTN & 188 & 53 & 28.70 & 71.3 & & Cross-sectional \\
\hline Rajan et al, $2019^{55}$ & India & HTN & 200 & 56.6 & 40.5 & 59.5 & MCQ & Cross-sectional, Convenient S \\
\hline Kisokanth et al $2018^{46}$ & Sri Lanka & HTN & 424 & 60.4 & 41 & 59 & MCQ & Cross-sectional, SRS \\
\hline Obirikorang et al, $2018^{51}$ & Ghana & HTN & 300 & 63.6 & 62 & 38 & Y & $\begin{array}{l}\text { Cross-sectional, Simple } \\
\text { Random }\end{array}$ \\
\hline Bakhsh et al, $2017^{36}$ & Saudi Arabia & HTN & 211 & 51.8 & 60.2 & 39.8 & Y & Cross-sectional, \\
\hline Rajasekharan et al, $2015^{56}$ & India & DM & 290 & 47.9 & 60 & 40 & MCQ & Cross-sectional, Convenient S \\
\hline Metias et al, $2017^{67}$ & Canada & $\begin{array}{l}\text { DM \& } \\
\text { HTN }\end{array}$ & 791 & - & - & - & & Prospective Survey \\
\hline Bacha et al $2019^{35}$ & Ethiopia & HTN & 385 & 57.6 & 54.5 & 48.3 & & Cross-sectional, SRS \\
\hline Duque et al, $2018^{41}$ & Portugal & HTN & 163 & - & - & - & $\mathrm{O \& C}$ & $\begin{array}{l}\text { Cross-sectional, Simple } \\
\text { Random }\end{array}$ \\
\hline N IK and B VR, $2015^{48}$ & India & $\begin{array}{l}\text { DM \& } \\
\text { HTN }\end{array}$ & 100 & 59 & 73 & 27 & o & Retrospective cross sectional \\
\hline Parappilly et al, $2019^{17}$ & Colombia & $\begin{array}{l}\text { DM \& } \\
\text { HTN }\end{array}$ & 100 & 66.6 & 60 & 4 & MCQ & Cross-sectional, Convenient S \\
\hline Kaddumukasa et al, $2017^{45}$ & Uganda & HTN & 440 & 54 & - & - & & Cross-sectional, \\
\hline Lai et al, $2015^{49}$ & Taiwan & DM & 597 & 51.28 & - & - & & Retrospective, SRS \\
\hline Selcuk Ozkan et al, $2019^{58}$ & Turk & HTN & 336 & 53.2 & 39 & 61 & & Cross-sectional, consecutive $S$ \\
\hline
\end{tabular}

Abbreviations: C, closed ended; O, open ended; MCQ, multiple choice questions; Y, yes/no questions; SRS, systematic random sampling; S, sampling; DM, diabetes mellitus; HTN, hypertension; Np, not participated.

Table 2 Overall Knowledge of HTN and DM Patients Towards Stroke

\begin{tabular}{|c|c|c|c|c|}
\hline Study & Country & Disease Condition & Overall Good Knowledge of Stroke (\%) & Know Stroke Affects Brain (\%) \\
\hline Abate $A^{29}$ & Ethiopia & HTN & $18.30^{\mathrm{a}}$ & 21.6 \\
\hline Ehidiamen $^{43}$ & Nigeria & HTN & NR & 56.3 \\
\hline $\operatorname{Dar}^{39}$ & Pakistan & HTN & NR & 76 \\
\hline Weltermann ${ }^{65}$ & Germany & DM & $39.4^{\mathrm{a}}$ & $\begin{array}{l}\text { 50- Native } \\
25 \text {-Migrant }\end{array}$ \\
\hline Oparah $^{53}$ & Nigeria & DM \& HTN & 16.9 & NR \\
\hline Arisegi ${ }^{34}$ & Nigeria & DM \& HTN & 70.3 & 87.4 \\
\hline Amen $^{32}$ & Iraq & HTN & 4.4 & NR \\
\hline Ampiah $^{33}$ & Ghana & DM \& HTN & $79.16^{\mathrm{b}}$ & 68.30 \\
\hline Venkatesh $^{61}$ & India & HTN & 66.7 & NR \\
\hline
\end{tabular}

Notes: Associated factors: age <6| years, educated, urban residents, high income, ${ }^{b}$ married, employed, educated, NR-not reported.

Abbreviations: DM, diabetes mellitus; HTN, hypertension. 


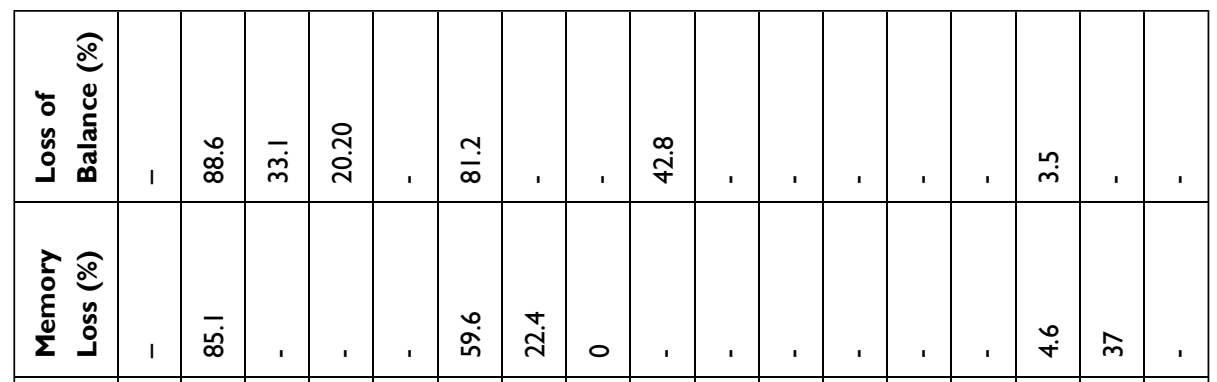

\begin{tabular}{|c|c|c|c|c|c|c|c|c|c|c|c|c|c|c|c|}
\hline 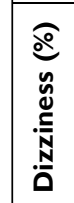 & 1 & 1 & ' & ' & ڤํ. & 卣 & $\begin{array}{l}\infty \\
\dot{q} \\
\dot{q}\end{array}$ & , & ' & , & , & $\frac{t}{\sigma}$ & $\stackrel{\circ}{\stackrel{\circ}{*}}$ & $\stackrel{\infty}{+}$ & $\bar{i}$ \\
\hline
\end{tabular}

\begin{tabular}{|c|c|c|c|c|c|c|c|c|c|c|c|c|c|c|c|}
\hline 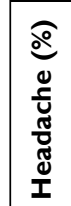 & 1 & 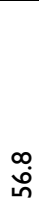 & $\stackrel{\text { సे }}{\text { in }}$ & $\hat{\ddot{\nu}}$ & ๕ & ఝి & $\underset{\text { di }}{\infty}$ & 0 & $f$ & ' & $\hat{\dot{q}}$ & $\widehat{\hat{\infty}}$ & $\stackrel{n}{m}$ & $\stackrel{\sim}{m}$ & $\stackrel{t}{\circ}$ \\
\hline
\end{tabular}

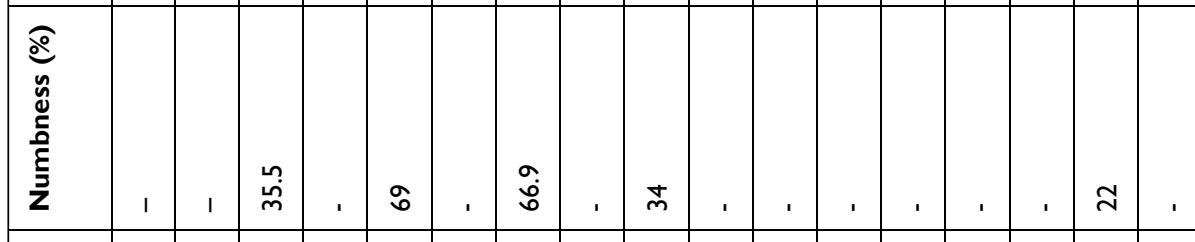

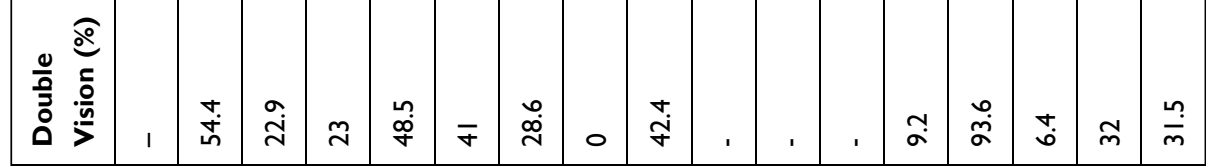




\begin{tabular}{|c|c|c|c|c|c|c|c|c|c|c|c|c|c|c|c|c|c|c|c|c|c|}
\hline 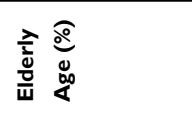 & 1 & $\stackrel{m}{m}$ & . & . & స్ & & & & & $\underset{\mathfrak{g}}{\mathscr{q}}$ & . & & 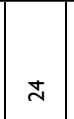 & $\stackrel{n}{\stackrel{n}{\rho}}$ & $\stackrel{m}{m}$ & & & & & $\hat{m}$ & $\simeq$ \\
\hline 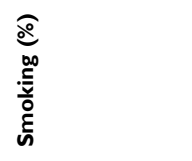 & 1 & స్త & $\stackrel{\infty}{m}$ & - & $\stackrel{\circ}{\infty}$ & & $\mid \begin{array}{c}\infty \\
\stackrel{\infty}{\sim}\end{array}$ & $\dot{m}$ & $\stackrel{+}{\dot{m}}$ & $\pi$ & . & $\stackrel{m}{q} \underset{q}{q}$ & $=$ & $\stackrel{n}{\stackrel{n}{m}}$ & $\hat{\dot{o}}$ & $\stackrel{\stackrel{\alpha}{\underline{j}}}{ }$ & oे & هُ & & $\hat{\tilde{y}}$ & \& \\
\hline 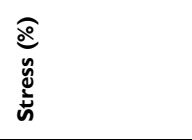 & 1 & $\overline{\dot{m}}$ & 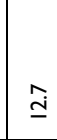 & . & $\underset{\infty}{\tilde{\infty}}$ & & & & & & . & & & & 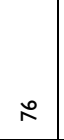 & $\bar{\sigma}$ & 乡 & & & $\hat{\underline{m}}$ & $\approx$ \\
\hline 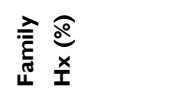 & 1 & $\overline{\underline{s}}$ & . & . & $\stackrel{\circ}{\circ}$ & $\stackrel{\stackrel{\circ}{\infty}}{\stackrel{\infty}{0}}$ & & $\simeq$ & & 离 & . & & น̊ & & $\widetilde{q}$ & & $\stackrel{a}{\dot{T}}$ & aे & & $\bar{o}$ & \\
\hline $\begin{array}{l}\text { @̊ } \\
\text { Q̊ }\end{array}$ & 1 & . & $\stackrel{\circ}{\circ}$ & . & $\mid \begin{array}{l}\infty \\
0 \\
0\end{array}$ & 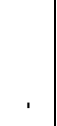 & $\bar{\sigma}$ & $\simeq$ & & $\begin{array}{c}\pi \\
\tilde{\sigma}\end{array}$ & . & & - & & & & & $\stackrel{n}{\sim}$ & & & \\
\hline 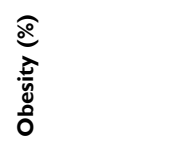 & $\bar{i}$ & . & $\underline{\underline{g}}$ & . & 㫟 &. & & $\simeq$ & & œ & . & 㝏 & $\approx$ & 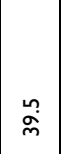 & & & హे & $\overline{\underline{\mathrm{I}}}$ & & $\hat{i}$ & $\stackrel{2}{\sim}$ \\
\hline 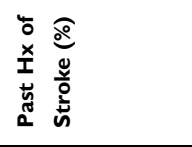 & 1 & . & . & . & 今. &. & & $\stackrel{N}{ }$ & & & . & $\stackrel{\rho}{\stackrel{\rho}{m}}$ & นn & & & & $\stackrel{\sim}{\infty}$ & & & & $m$ \\
\hline 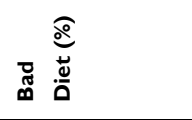 & 1 & . & $\stackrel{\circ}{\circ}$ & . & \begin{tabular}{|c}
$\stackrel{\circ}{\infty}$ \\
$\infty$
\end{tabular} &. & & & & $\hat{\infty}$ & . & & & $\mathscr{\gamma}$ & $\pi$ & & & $\underset{\infty}{+}$ & & & \\
\hline 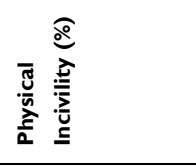 & $\frac{0}{N}$ & . & . & . & $\bar{i}$ & . & , & . & & \begin{tabular}{|c}
$\infty$ \\
$\infty$ \\
$\infty$ \\
\end{tabular} & . & $\begin{array}{l}m \\
\tilde{r} \\
\end{array}$ & in & $\stackrel{n}{\stackrel{n}{\simeq}}$ & \pm & & $\hat{\alpha}$ & $\stackrel{\stackrel{n}{\sim}}{\wedge}$ & & $\begin{array}{l}\underline{n} \\
\underline{0} \\
\end{array}$ & 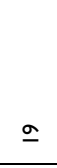 \\
\hline 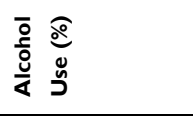 & $\underline{\widehat{\omega}}$ & . & $\stackrel{\leftrightarrow}{q}$ & . & $\overline{\dot{f}}$ & . & 主 & $\tilde{m}$ & & $\stackrel{\infty}{\underset{N}{N}}$ & . & $\dot{m}$ & . & $\overline{\dot{g}}$ & 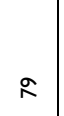 & $\hat{a}$ & $\hat{\text { sj }}$ & $\stackrel{\circ}{=}$ & & $\hat{f}$ & \\
\hline 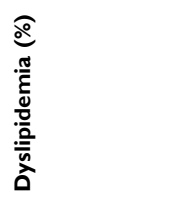 & 1 & . & . & . & $\overline{\dot{\infty}}$ & . & $\stackrel{\leftrightarrow}{+}$ & \pm & $\hat{i}$ & $\hat{\infty}$ & . & 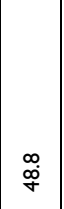 & $m$ & & 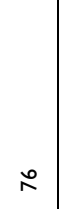 & & $\sigma^{m}$ & $\circ$ & & $\stackrel{ \pm}{\sim}$ & \\
\hline$\stackrel{\S}{\varrho}$ & , & 吕 & $\stackrel{m}{n}$ & 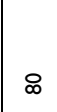 & $\stackrel{n}{n}$ & , & $\stackrel{\circ}{i}$ & ț & 岁 & $\stackrel{n}{ת}$ & . & q & $\frac{n}{6}$ & . & $\approx$ & $\tilde{\Xi}$ & $\overline{\dot{\infty}}$ & $\hat{\infty}$ & $\circ$ & $\stackrel{ \pm}{\stackrel{ \pm}{I}}$ & $n$ \\
\hline$\frac{\text { @̊ }}{z}$ & $\stackrel{\circ}{m}$ & $\stackrel{\infty}{\text { in }}$ & fे & in & $\approx$ & 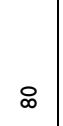 & $\stackrel{n}{\stackrel{n}{\Lambda}}$ & $\stackrel{2}{2}$ & $\underset{\sigma}{+}$ & $\sigma$ & . & 官 & $\infty$ & & $\hat{\infty}$ & $\underset{m}{\stackrel{\dagger}{m}}$ & $\widehat{\infty}$ & $\stackrel{+}{\vec{N}}$ & $\bar{m}$ & $g$ & ळ \\
\hline 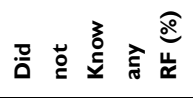 & $R$ & . & in & m. & & . & & . & $\tilde{\sim}$ & . & . & ( & $\underline{n}$ & $\stackrel{\sim}{\sim}$ & . & & \pm & $\overline{\mathrm{\rho}}$ & & & \\
\hline 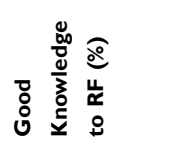 & \pm & \begin{tabular}{|c}
0 \\
$\infty$ \\
$m$
\end{tabular} & 紫 & . & & . & & . & $\stackrel{n}{\alpha}$ & $\begin{array}{l}\stackrel{\circ}{0} \\
\infty\end{array}$ & $\stackrel{m}{q}$ & & & 总 & . & 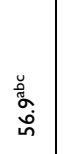 & & $\underset{ \pm}{ \pm}$ & & & \\
\hline 害 & 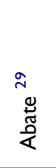 & 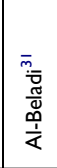 & 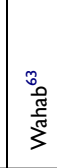 & 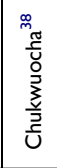 & 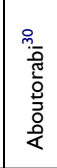 & 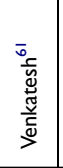 & 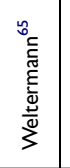 & 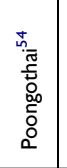 & 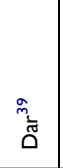 & 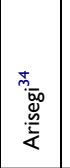 & 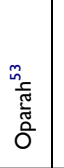 & 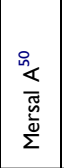 & 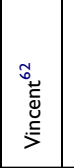 & 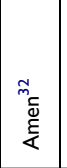 & 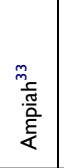 & 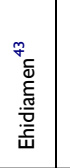 & 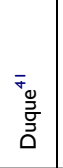 & 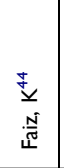 & 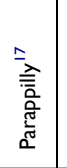 & $\begin{array}{l}\hat{o}_{\tilde{u}} \\
\stackrel{\tilde{m}}{\tilde{\omega}} \\
\Sigma\end{array}$ & 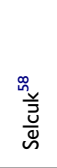 \\
\hline
\end{tabular}


Table 5 Knowledge Towards the Prevention of Stroke

\begin{tabular}{|l|l|l|l|l|l|l|l|l|}
\hline Study & $\begin{array}{l}\text { Good Knowledge of } \\
\text { Prevention (\%) }\end{array}$ & $\begin{array}{l}\text { Know Stroke is } \\
\text { Preventable (\%) }\end{array}$ & $\begin{array}{l}\text { Avoid } \\
\text { Alcohol (\%) }\end{array}$ & $\begin{array}{l}\text { Avoid Fat } \\
\text { Intake (\%) }\end{array}$ & $\begin{array}{l}\text { Smoking } \\
\text { Cessation (\%) }\end{array}$ & $\begin{array}{l}\text { Physical } \\
\text { Activity (\%) }\end{array}$ & $\begin{array}{l}\text { Weight } \\
\text { Reduction (\%) }\end{array}$ & $\begin{array}{l}\text { Vegetable } \\
\text { Use (\%) }\end{array}$ \\
\hline Venkatesh $^{61}$ & - & - & 9.5 & 45.7 & 5.7 & 42.8 & 5.7 & 37.1 \\
\hline Dar $^{39}$ & - & 85.4 & - & 40.4 & 85.2 & 49 & - & 76 \\
\hline Arisegi $^{34}$ & $90.8{ }^{\text {a }}$ & - & 77.8 & 88.7 & 78.6 & 88.3 & 87.9 & 87 \\
\hline Parappilly $^{17}$ & - & - & - & 57 & - & 51 & - & 52 \\
\hline Ampiah $^{33}$ & - & 82 & - & - & - & - & - & - \\
\hline
\end{tabular}

Notes: Associated factors: ${ }^{\text {a }}$ educational and employment status.

were knowing whether a stroke is preventable. ${ }^{33,39}$ Furthermore, four studies investigated and reported regular physical exercise (49-88\%), avoiding fat intake (40-89\%), and frequent use of vegetables (37-87\%). ${ }^{17,34,39,61}$ Likewise, three studies reported whether subjects had knowhow of smoking cessation (6-85\%) as prevention of stroke. ${ }^{34,39,61}$ Avoiding alcohol use and weight reduction were also revealed by studies as shown in Table 5 .

\section{Prevention Practices Towards Stroke}

One of the prevention strategies of stroke emergency is prestroke LMP which is supported by many pieces of evidence. ${ }^{70}$ In this review, we extracted data from primary studies that were done related to LMP. In doing so, 29 studies were reviewed to generalize the trends of stroke prevention practices.

As a result, seven studies reported 2.4\% (South Africa) $)^{52}$ to $72 \%$ (Ghana) $)^{51}$ prevalence of good LMP among the respondents. But only three studies were reporting more than $50 \%$ good practice. ${ }^{51,59,61}$ When asked what actions have you ever practice to maintain their body and to prevent the incidence of stroke, the majority of the studies reported sub-optimal practice; nineteen studies reported for smoking cessation $(>50 \%)$, fourteen studies for avoiding alcohol (60-90\%), nine studies for avoiding added salt (57-95\%), five studies for vegetable use (60 $100 \%$ ), four studies for regular home/facility BP or blood glucose (BG) checking (57-77\%), three studies for reducing fat intake (59-95\%). However, the practice of regular physical exercise was too low. Similarly, weight reduction practice was low as shown in Table 6.

\section{Discussion}

To the reviewer's knowledge, this systematic review has been the first paper synthesized regarding knowledge and prevention practices of stroke amongst hypertensive and diabetic patients. To undergo the review, the results of 42 existing articles were collected. The reported results were variable in between the studies. This might be due to the difference in the time course of the conducted studies and/or the variability in the socioeconomic status of the study population/study setting/. Irrespective of this, the overall results of the review show that the prevalent level of knowledge and LMP towards stroke was limited. Almost $40-80 \%$ of subjects were not aware of what a stroke is and the organ to be affected. ${ }^{29,43,65}$ Obviously, individuals with HTN and/or DM are at greater risk of suffering stroke. ${ }^{8,71}$ Hence, it is expected to be familiar with stroke and its warning signs.

The present review depicted that there was low awareness of the clinical signs and symptoms of a stroke. ${ }^{32,53}$ In contrast, two studies showed better knowledge of the signs/symptoms of a stroke. ${ }^{33,34}$ Even if suboptimal knowledge was found in other signs/symptoms, more than $50 \%$ of respondents did not know the exact double vision and severe headache as symptoms of a stroke in the majority of the reviewed studies. Severe headache, ${ }^{72}$ and double vision/diplopia are major clinical presentations that should be identified as warning symptoms of a stroke. $5,73,74$ So far, the reviewed studies reported $15-77 \%$ of patients with no knowledge of any of the mentioned warning signs of stroke as shown in Table 3.

It is important to note that left to unaware these vulnerable groups to the warning signs will come up with complicated medical situations up on attacked with stroke events. This is because not identifying the early warning signs leads to a prehospital delayed. The main reason for stroke victims too late hospital presentation was considered as their inability to recognize the symptoms of stroke, waiting for the sign to abate by itself, and eventually lack of awareness of the advantages that could be obtained from early treatment of thrombolysis. ${ }^{14-16}$ On the contrary, having previous information to this able to increased patient's early hospital arrival by 


\begin{tabular}{|c|c|c|c|c|c|c|c|c|c|c|c|c|c|c|c|c|c|c|c|c|c|c|}
\hline 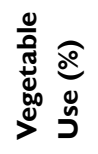 & ડે & $\therefore$ & 1 & $\frac{\infty}{\sigma}$ & . & ' & $\hat{n}$ & , & & ' & ' & $m$ & & , & ' & . & ' & ' & ' & ํㅡ & बू. & \\
\hline 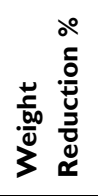 & 1 & 1 & 1 & $\begin{array}{l}\infty \\
\stackrel{\infty}{\oplus} \\
\dot{m}\end{array}$ & ' & ' & ' & ' & $\vec{i}$ & ' & $\frac{\sigma}{\bar{\sigma}}$ & ' & , & ' & in & ' & ' & @ं & ' & , & & 8 \\
\hline 总 & గે & $q$ & กี่ & . & ' & $\stackrel{\infty}{\sim}$ & ' & ' & ${ }_{\infty}^{m}$ & $\underline{\Xi}$ & $\underline{0}$ & ळ & $\sigma$ & 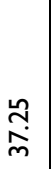 & $f$ & $\stackrel{n}{\stackrel{n}{\sim}}$ & $\begin{array}{l}\stackrel{2}{\tilde{N}} \\
\underline{\underline{\Theta}}\end{array}$ & ก่ં & $\sigma$ & ' & , & $\stackrel{m}{i}$ \\
\hline
\end{tabular}

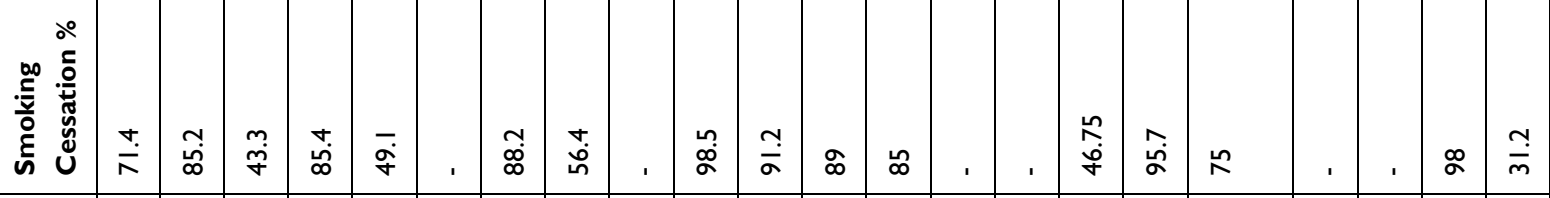

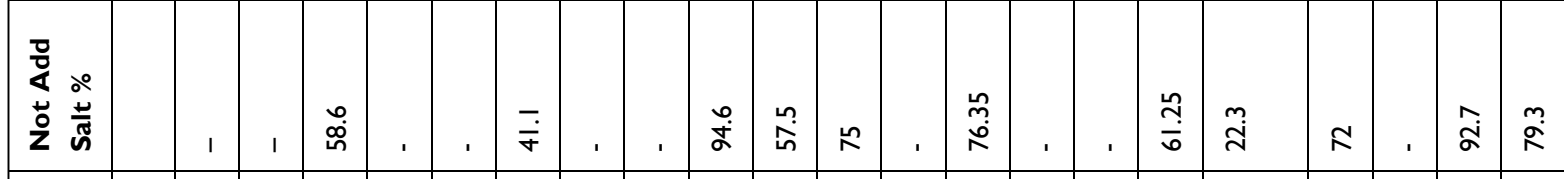

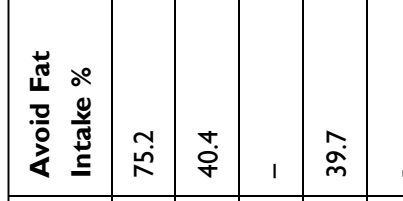

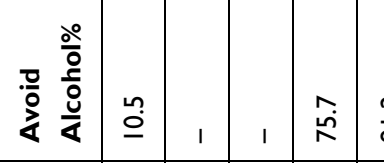

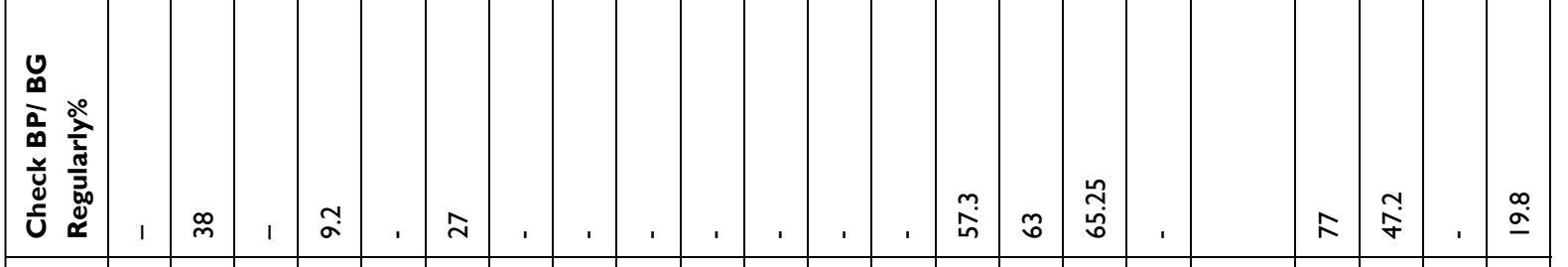

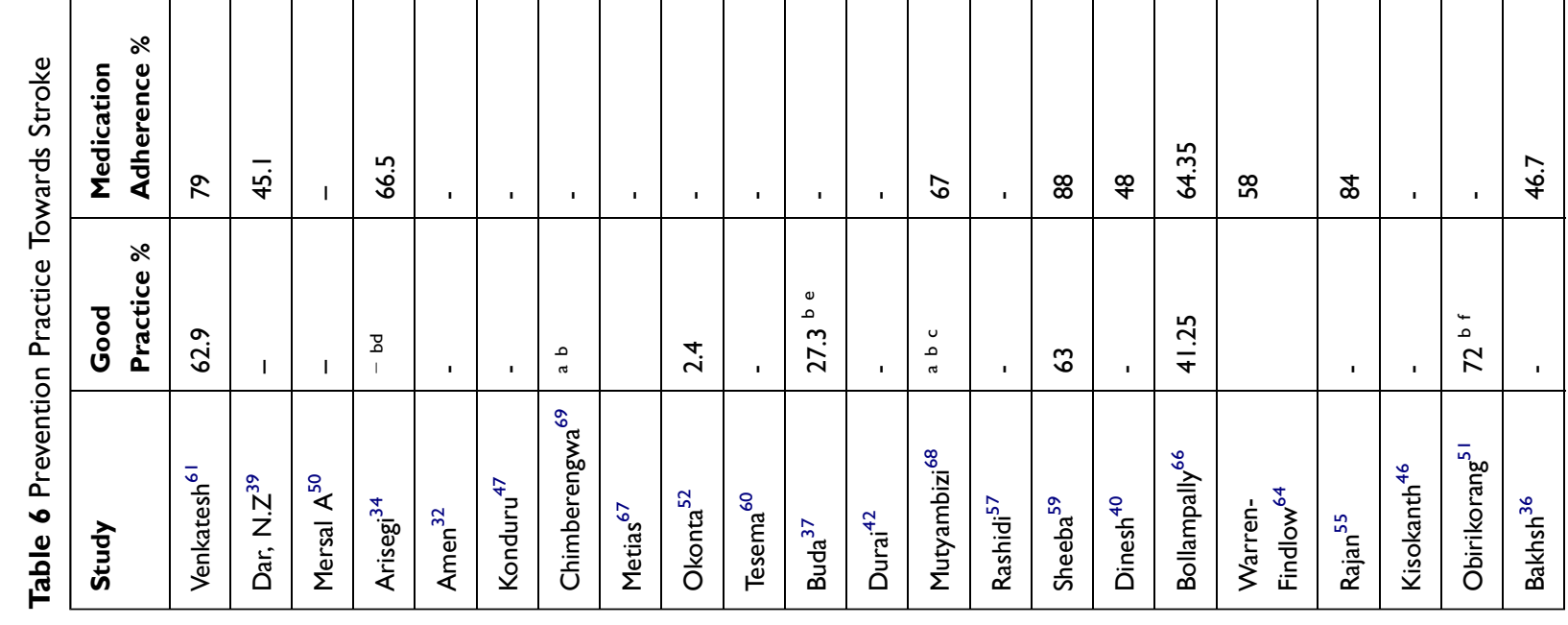




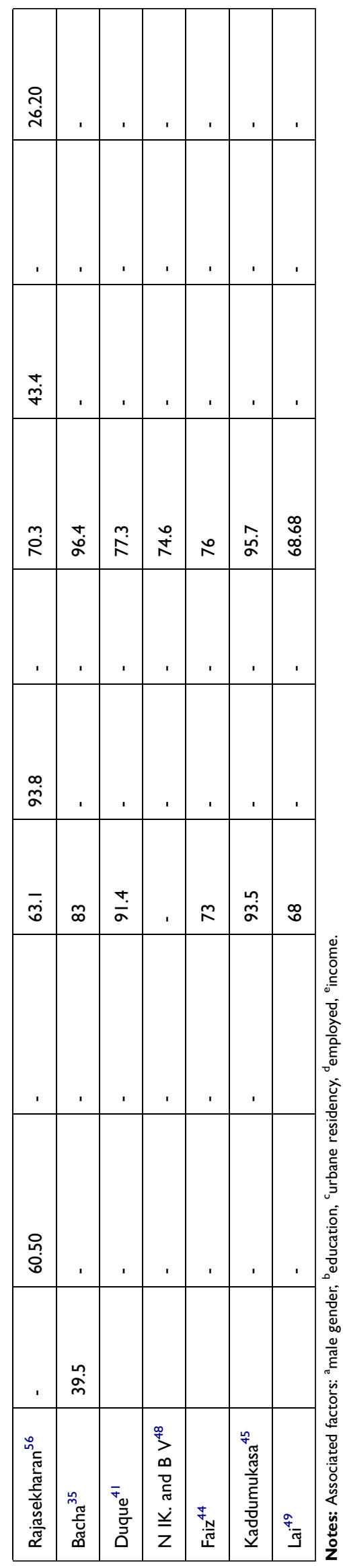

10-fold thereby enhanced thrombolysis eligibility. ${ }^{75}$ Therefore, creating awareness of the sign and treatment of stroke has a paramount advantage to enhance the need for a rapid medical call. ${ }^{74-76}$

Years ago, many countries were launching an effective Face Arm Speech Time (FAST) campaign that can have a positive implication to increase public awareness to the warning signs of stroke and thereby to promote immediate response; it aimed to raise the need for rapid medical call whenever anyone manifest early warning signs and symptoms of stroke, ie, facial deviation, arm weakness, and speech disturbance. Their intention was not only to these signs of stroke but as far as possible to familiarize people even to other signs and symptoms of a stroke. Because the clinical manifestations may not be always similar in all individuals. $^{74,77-81}$ Therefore, this review also suggests such educational strategies should be reconsidered and planned in the future.

Stroke awareness is necessary not only to the clinical signs and symptoms but also to the risk factors and prevention as well. The ultimate objective of conducting population awareness is to increase the trend of abstaining from those contributing lifestyle risk factors. However, almost all the reviewed studies stated that the HTN and/ or DM patients' level of recognition to the potential relation of stroke and risk factors has been low so that they were recommending educational programs/campaigns to be held as an important concern of the national health policies. ${ }^{29-31,38,39,43,50,53,54,65}$

Since stroke is multi-factorial, people especially risky individuals should be conscious enough to measure their level of exposure and to be away from those risk factors. Risk factor knowledge begins with the identification of the current own medical conditions. Surprisingly, $64.6 \%$ to $94.6 \%$ of the hypertensive $\mathrm{e}^{29,43}$ and more than $78 \%$ of the diabetic patients ${ }^{65}$ were found that they did not know whether their own medical condition is the cause of a stroke as shown in Table 4.

By far the most cited risk factor of stroke in the majority of the reviewed studies was HTN. ${ }^{30,31,34,38,39,41,50,54,58,61,62}$ Though HTN is the major, it is not the only risk of stroke; other risk factors should be identified well. Some studies reported that more than $50 \%$ of the respective respondents with no knowledge to at least one risk factor of stroke. ${ }^{29,44,63}$ Of course, being hypertensive and/or hyperglycemic contributes to stroke events by itself; their effect is much accelerated whenever other modifiable lifestyle factors (ie, smoking, excessive alcohol use, physical inactivity/sedentary life, 
obesity, high cholesterol, and habit of diet) are poorly managed. $^{70,82-85}$ However, the result of this review revealed that more than $85 \%$ of the sampled participants did not consider the above-listed behavioral factors as the risk of stroke. $38,44,62,63,65,86$

Interestingly, stress that was highlighted in many of the reviewed studies; $12.7 \%{ }^{63}$ to $96 \%{ }^{41}$ of the subjects consider it exactly as the risk of stroke. Noticeably, stressful life events increase 2-3 times to the emergence of stroke by increasing excessive sympathomimetic activity and other different postulated mechanisms. ${ }^{87-90}$ So, people at risk of stroke should identify and be away from any psychologically stressful situations. The review also found a low level of knowledge of the non-modifiable risk factors as if a stroke is highly associated with older age, family, and previous history of stroke. $^{31-34,44,50,54,58,62}$ Therefore, it should be of great concern to healthcare providers that so many patients do not understand the cause of stroke and have a limited understanding of basic stroke prevention methods.

Another important finding worth of highlighted in this review was LMP as the primary prevention (PP) of stroke. Though stroke is preventable primarily by avoiding or reducing the contributing risk factors, ${ }^{70}$ the result of this review shows that more than $60 \%$ of the vulnerable individuals did not strive to prevent stroke and other cardiovascular complications. ${ }^{35,37,52,61,66}$ Take for example smoking; the pattern of smoking habit contributes 2-4 times the likelihood of developing stroke as compared to non-smokers. ${ }^{91,92}$ Nonetheless, with this review, 50-70\% of subjects were found to be smokers with a double burden to their medical condition in some studies. ${ }^{32,36,40,50}$ This could amplify the rate of stroke in risky individuals unless immediate action is taken. ${ }^{84,85}$

Although its detailed biological mechanism is not clearly stated, evidence suggests physical activity (3-5 days/week) to reduce the likelihood of a stroke event. ${ }^{93-95}$ Unfortunately, subjects of the review level of engagement to practice regular physical exercise were found to be low. More than $70 \%$ of papers stated $<50 \%$ of the respondents were striving to do regular physical exercise as shown in Table 6. Sedentary life has been the leading cause of mortality in the world; it increases $20-30 \%$ risk of all causes of death. Hence, WHO proposed in raising awareness of physical activity and to be a key component in the prevention of non-communicable diseases including stroke. ${ }^{96}$

Moreover, weight reduction was reported as a poor practice by many reviewed studies. ${ }^{34,37,52,64}$ Overweight and obesity are important public health problems associated with many serious medical conditions including stroke. Recent American College of Cardiology and American Heart Association (ACC/AHA) guidelines recommend weight reduction as $\mathrm{PP}$ of stroke and other cardiovascular complications. ${ }^{97}$ Alternatively, a cohort study also suggests that not only weight gain but also weight loss more than $5 \%$ should be emphasized to the increased incidence rate of Transient ischemic attack (TIA). ${ }^{98}$ Therefore, weight management to be optimal to prevent a stroke rate.

The trend of alcohol reduction practice was good in most studies but a single study in India showed too low (10.5\%). ${ }^{61}$ This worldwide prevalent practice has been established as a risk factor for stroke disease principally hemorrhagic stroke since alcohol affects the coagulation pathway and cerebral vessel integrity. ${ }^{99}$ In fact, excessive alcohol use is a risk factor of stroke events, low to moderate alcohol intake has advantages to reduce the incidence of ischemic stroke. ${ }^{99-101}$ Regardless of this controversy alcohol is not that much recommended to be used especially in risky individuals.

Another point of discussion was medication adherence, which was reported $45-90 \%$ adherence to the prescribed antidiabetic and hypertensive medications as shown in Table 6. Even if they did not feel any problem, 55-77\% of them were regularly checking their BP and/or BG either inhome or health facilities as was reported by the reviewed studies. $^{40,55,57,59}$ This is good practice that should be advocated more for early prevention of HTN and DM-associated emergence of stroke. Because the most plausible reasons for stroke events have been uncontrolled BP and BG. ${ }^{1,4,9}$

So far, dietary conditions, ie, fat, salt, and vegetable use should have to be emphasized for PP of stroke as if there has been a suboptimal practice in few reviewed studies. ${ }^{102}$ Evidence showed that bad nutritional formula is considered as the risk of stroke, especially in high-risk populations. Early prevention of stroke and other cardiovascular complications starts from adjusting the day-today dietary conditions. For example, habiting the Mediterranean diet, high in olive oil, whole grains, fruits, vegetables, and legumes, and low in cholesterol/saturated fat is advised as prevention of stroke. ${ }^{103-105}$ Habiting these diets can reduce the stroke rate by up to $40 \%$ or more in high-risk patients. ${ }^{104}$ Hence, awareness to apply diet-based stroke prevention is necessary to these populations.

Finally, significantly associated sociodemographic factors of stroke knowledge and practice were systematically reviewed. As a result, educational level, 29,31,32,34,43,53,63 urban residency, ${ }^{29,33,63}$ employment, ${ }^{33,34}$ age $<60$ 
years, ${ }^{29,32,34,63,65}$ monthly income ${ }^{29}$ and marital status ${ }^{30}$ were statistically associated with good level of stroke knowledge. Moreover, male gender in Nigeria ${ }^{43}$ and female in Iraqi $^{32}$ was found to be strongly associated sole predictor variable of stroke knowledge. Similarly, LMP was affected with these independent sociodemographic factors; male gender, ${ }^{10,35}$ higher educational level, ${ }^{34,35,37,51,69}$ urban residency, ${ }^{35}$ income, $^{37}$ employment ${ }^{34}$ and marital status. ${ }^{51}$ Meanwhile, the elderly and comorbidity were the preventive factors of good stroke prevention practice. ${ }^{37}$ The main highlighted reason for the high level of knowledge and practice to these subgroups was due to the sufficient and easy accessibility of the different information sources to them.

\section{Limitation}

Despite this review has strong parts like critically apprizing and compiling the results of the published articles systematically, some other limitations that we could not avoid may be here. The limitations might be obtained from the reviewed studies; some data were not complete; their data collection questioneers were not similar in studies that could reduce the generalizability of this review. Additionally, all the reviewed articles were published in journal cites; unpublished studies could not be accessed. So far, to show the result quantitatively, the pooled estimated value was not attempted to be calculated because of diverse outcomes and the insufficient data reported in the studies.

\section{Conclusion}

It is clear from the above discussion that knowledge and prevention practice of stroke amongst hypertensive and diabetic patients has been low. The reported level of knowledge in high-risk populations towards the general concepts, warning signs, risk factors, and prevention methods of stroke was very low. There is also suboptimal lifestyle modification practice to prevent stroke. Especially the trend of regular physical activity and weight reduction practice was relatively low. This inadequate knowledge and prevention practice were associated with elderly, educationally low, economically poor, rural residents, unmarried, unemployed, and comorbid populations. Finally, the review highlights the importance of increasing public awareness about stroke symptoms, risk factors, and prevention strategies as well.

\section{Abbreviations}

ACC/AHA, American College of Cardiology and American Heart Association; AHA/ASA, American Heart Association and American Stroke Association; BG, Blood
Glucose; DALYS, Disability-Adjusted Life Years; DM, Diabetes Mellitus; HTN, Hypertension; LMP, Lifestyle Modification Practice; NIH, National Institute of Health; PP, Primary Prevention; SSA, Sub-Saharan African; TIA, Transient Ischemic Attack; tPA, Tissue Plasminogen Activator; WHO, World Health Organization.

\section{Acknowledgment}

The authors would like to thank their colleagues who gave constructive feedback on the completion of this paper.

\section{Funding}

We did not receive specific funding for this work.

\section{Disclosure}

The authors report no conflicts of interest in this work.

\section{References}

1. Feigin VL, Nichols E, Alam T, et al. Global, regional, and national burden of neurological disorders, 1990-2016: a systematic analysis for the Global Burden of Disease Study 2016. Lancet Neurology. 2019;18(5):459-480. doi:10.1016/ S1474-4422(18)30499-X

2. Powers WJ, Rabinstein AA, Ackerson T, et al. Guidelines for the early management of patients with acute ischemic stroke: 2019 update to the 2018 guidelines for the early management of acute ischemic stroke: a guideline for healthcare professionals from the American Heart Association/American Stroke Association. Stroke. 2019;50(12): e344-e418.

3. WHO. The top 10 causes of death; 2016. Available from: https:// www.who.int/news-room/fact-sheets/detail/the-top-10-causes-ofdeath. Accessed August 4, 2021.

4. Namale G, Kamacooko O, Kinengyere A, et al. Risk factors for hemorrhagic and ischemic stroke in sub-Saharan Africa. $J$ Trop Med. 2018;2018:1-11. doi:10.1155/2018/4650851

5. Fekadu G, Chelkeba L, Kebede A. Risk factors, clinical presentations and predictors of stroke among adult patients admitted to stroke unit of Jimma university medical center, south west Ethiopia: prospective observational study. BMC Neurol. 2019;19 (1):183. doi:10.1186/s12883-019-1412-5

6. Owolabi MO, Sarfo F, Akinyemi R, et al. Dominant modifiable risk factors for stroke in Ghana and Nigeria (SIREN): a case-control study. Lancet Global Health. 2018;6(4):e436-e446. doi:10.1016/S2214-109X(18)30002-0

7. Zhang F-L, Guo Z-N, Wu Y-H, et al. Prevalence of stroke and associated risk factors: a population based cross sectional study from northeast China. BMJ Open. 2017;7(9):e015758. doi:10.1136/bmjopen-2016-015758

8. Habibi-koolaee M, Shahmoradi L, Niakan Kalhori SR, Ghannadan H, Younesi E. Prevalence of stroke risk factors and their distribution based on stroke subtypes in gorgan: a retrospective hospital-based study-2015-2016. Neurol Res Int. 2018;2018:548.

9. Onwuegbuzie GA, Reng RS. Diabetes as a risk factor for ischemic stroke in a tertiary health care center, Abuja. New Nigerian J Clin Res. 2018;7(11):16.

10. Owolabi L, Nagode M, Ibrahim A, Uloko A, Gezawa I, Datti M. Stroke in patients with diabetes mellitus: a study from North Western Nigeria. Afr Health Sci. 2016;16(3):781-789. doi:10.4314/ahs.v16i3.19 
11. Sharif F, Ghulam S, Sharif A. Prevalence of risk factors associated with stroke. Pakistan Heart J. 2019;52(1):2-85.

12. Ghimire MR, Soti B, Prajapati S, Thapa L, Ghimire S, Shrestha S. Risk factors associated with stroke among the clients attending Neuro and Allied Clinic, Bhairahawa. Stroke J. 2019;3 (1):1-8.

13. Saeedi P, Petersohn I, Salpea P, et al. Global and regional diabetes prevalence estimates for 2019 and projections for 2030 and 2045: results from the International Diabetes Federation Diabetes Atlas. Diabetes Res Clin Pract. 2019;157:107843. doi:10.1016/j. diabres.2019.107843

14. Arulprakash N, Umaiorubahan M. Causes of delayed arrival with acute ischemic stroke beyond the window period of thrombolysis. J Family Med Primary Care. 2018;7(6):1248. doi:10.4103/jfmpc. jfmpc_122_18

15. Fladt J, Meier N, Thilemann S, et al. Reasons for prehospital delay in acute ischemic stroke. $J$ Am Heart Assoc. 2019;8(20): e013101. doi:10.1161/JAHA.119.013101

16. Mahmoud Hewedi K. Pre-hospital and in hospital delays after the onset of acute ischemic stroke in a sample of Egyptian patients. Al-Azhar Med J. 2019;48(4):311-322. doi:10.21608/ amj.2019.64940

17. Parappilly BP, Field TS, Mortenson WB, Sakakibara BM, Eng JJ. Determinants influencing the prestroke health behaviors and cardiovascular disease risk of stroke patients: a Cross-Sectional Study. J Stroke Cerebrovascular Dis. 2019;28(6):1509-1518. doi:10.1016/j.jstrokecerebrovasdis.2019.03.015

18. Karkout RA, Shamaa O, Al Ammour ST, Abd Elmaged RS, Al Kassar LA, Al Ali MN. Knowledge about stroke among adults in Sharjah, United Arab Emirates. Int J Res Med Sci. 2019;7 (10):3632. doi:10.18203/2320-6012.ijrms20194194

19. Wan L-H, Zhao J, Zhang X-P, et al. Stroke prevention knowledge and prestroke health behaviors among hypertensive stroke patients in mainland China. $J$ Cardiovascular Nursing. 2014;29 (2):E1-E9. doi:10.1097/JCN.0b013e31827f0ab5

20. Sowtali SN, Harith S, Mohamed M, Yusoff DM. Stroke knowledge level among stroke patients admitted to hospital Raja Perempuan Zainab II, Kelantan, Malaysia. J Exp Stroke Transl Med. 2016;9(1):1-11.

21. Indira Kumari N, Veera Raghavulu B. Risk factor assessment of stroke and its awareness among stroke survivors: a retrospective study. Int J Res Health Sci. 2015;3(1):140-145.

22. Alzahrani FA, Alenzy AE, Alghamdi AM. Stroke in Tabuk, KSA: awareness and prevalence of risk factors. Life Sci J. 2019;16 (6): 256

23. Ojike N, Ravenell J, Seixas A, et al. Racial disparity in stroke awareness in the US: an analysis of the 2014 National Health Interview Survey. J Neurol Neurophysiol. 2016;7(2). doi:10.4172/ 2155-9562.1000365.

24. Sadeghi-Hokmabadi E, Vahdati SS, Rikhtegar R, Ghasempour K, Rezabakhsh A. Public knowledge of people visiting Imam Reza hospital regarding stroke symptoms and risk factors. BMC Emerg Med. 2019;19(1):36. doi:10.1186/ s12873-019-0250-5

25. Karnad A, Pannelay A, Boshnakova A, Lovell AD, Cook RG. Stroke prevention in Europe: how are 11 European countries progressing toward the European Society of Cardiology (ESC) recommendations? Risk Manag Healthc Policy. 2018;11:117. doi:10.2147/RMHP.S163439

26. Network E. PRISMA (2009) checklist for reporting systematic reviews and meta-analyses.. J ASEAN Federation Endocrine Societies. 2015;30(2):196.

27. Health NIo. National Institutes of Health Quality Assessment tool for Observational Cohort and Cross-Sectional Studies: National Institutes of Health. 2016.
28. Boateng D, Wekesah F, Browne JL, et al. Knowledge and awareness of and perception towards cardiovascular disease risk in sub-Saharan Africa: a systematic review. PLoS One. 2017;12:12. doi:10.1371/journal.pone.0189264

29. Abate AT, Bayu N, Mariam TG. Hypertensive patients' knowledge of risk factors and warning signs of stroke at Felege Hiwot Referral Hospital, Northwest Ethiopia: a cross-sectional study. Neurol Res Int. 2019;2019:1-7. doi:10.1155/2019/8570428

30. Aboutorabi-Zarchi M, Jam Ashkezari S, Gholami S, RazaviRatki SK, Namiranian N. Is Knowledge of type 2 diabetic patients about stroke acceptable - a cross sectional study. Iranian J Diabetes Obesity. 2019;11(1):22-27.

31. Al-Beladi B, Khulood M, Al O, Mesed A, Nafea R, Mosleh H. Awareness of stroke among diabetic and hypertensive patients at King Fahad hospital in al-madinah. KSA. 2018;2016:5.

32. Amen MR. Assessment of hypertensive patients' knowledge about lifestyle risk factors and warning signs of stroke. J Contemporary Med Sci. 2016;2(5):28-32.

33. Ampiah PK. 2018 knowledge of stroke among hypertensive-diabetic patients at the national diabetes management and research centre of Korle-bu teaching hospital in Ghana. J Prevent Rehabilitative Med. 2018;1(1):46-62.

34. Arisegi SA, Awosan KJ, Oche MO, Sabir AA, Ibrahim MT. Knowledge and practices related to stroke prevention among hypertensive and diabetic patients attending Specialist Hospital, Sokoto, Nigeria. Pan African Med J. 2018;29(1):1-17. doi:10.11604/pamj.2018.29.63.13252

35. Bacha D, Abera H. Knowledge, Attitude and self-care practice towards control of hypertension among hypertensive patients on follow-up at St. Paul's Hospital, Addis Ababa. Ethiop J Health Sci. 2019;29:4.

36. Bakhsh LA, Adas AA, Murad MA, et al. Awareness and knowledge on hypertension and its self-care practices among hypertensive patients in Saudi Arabia. Ann Int Med Dent Res. 2017;2(5). doi:10.21276/aimdr.2017.3.5.ME13.

37. Buda ES, Hanfore LK, Fite RO, Buda AS. Lifestyle modification practice and associated factors among diagnosed hypertensive patients in selected hospitals, South Ethiopia. Clin Hypertension. 2017;23(1):26. doi:10.1186/s40885-017-0081-1

38. Chukwuocha I, Anyanwu A, Nwazor E. Awareness of stroke among subjects with diabetes mellitus attending a tertiary diabetes outpatient clinic in South-East Nigeria. Int $J$ Endocrinol Metab Disord. 2018;4(1):364.

39. Dar NZ, Khan SA, Ahmad A, Maqsood S. Awareness of stroke and health-seeking practices among hypertensive patients in a tertiary care hospital: a cross-sectional survey. Cureus. 2019;11:5

40. Dinesh PV, Kulkarni AG, Gangadhar NK. Knowledge and self-care practices regarding diabetes among patients with Type 2 diabetes in Rural Sullia, Karnataka: a community-based, cross-sectional study. J Family Med Primary Care. 2016;5 (4):847. doi:10.4103/2249-4863.201176

41. Duque AS, Fernandes L, Correia AF, et al. Awareness of stroke risk factors and warning signs and attitude to acute stroke. Int Arch Med. 2015;8:8.

42. Durai V, Muthuthandavan AR. Knowledge and practice on lifestyle modifications among males with hypertension. Indian J Community Health. 2015;27(1):143-149.

43. Ehidiamen OF, Ehinwenma OJ. Awareness of stroke risk factors and warning symptoms amongst hypertensive patients in Benin city. Ann Med Health Sci Res. 2018;1:78.

44. Faiz KW, Sundseth A, Thommessen B, Rønning OM. Patient knowledge on stroke risk factors, symptoms and treatment options. Vasc Health Risk Manag. 2018;14:37. doi:10.2147/ VHRM.S152173 
45. Kaddumukasa M, Kayima J, Nakibuuka J, et al. Modifiable lifestyle risk factors for stroke among a high risk hypertensive population in Greater Kampala, Uganda; a cross-sectional study. BMC Res Notes. 2017;10(1):675. doi:10.1186/s13104-017-3009-7

46. Kisokanth G. Perceptions, attitudes and self-care practices on management of hypertension among hypertensive patients at teaching hospital, Batticaloa district, Sri lanka. Open Hypertens J. 2018;10(1):1-7. doi:10.2174/1876526201810010001

47. Konduru S, Ranjan A, Karthik SM, Shaik S, Vakkapatla LS. Assessment of diabetes related knowledge, attitude and practice among diabetics and non-diabetics using self prepared questionnaire for awareness of health promotion. Indian J of Pharm Pract. 2017;10:32-38. doi:10.5530/ijopp.10.1.8

48. Ik N, Vr B. Risk factor assessment of stroke \& its awareness among stroke survivors: a retrospective study. Int $J$ Res Health Sci. 2015;3(1):140-145.

49. Lai Y-J, Hu H-Y, Lee Y-L, Ku P-W. A retrospective cohort study on the risk of stroke in relation to a priori health knowledge level among people with type 2 diabetes mellitus in Taiwan. BMC Cardiovasc Disord. 2017;17(1):130. doi:10.1186/s12872-017-0568-4

50. Mersal FA, Tork HM. Stroke risk perception and its awareness among hypertensive patients in Qassim region Saudi Arabia. Majmaah J Health Sci. 2020;8(1):9-22. doi:10.5455/mjhs.2020.01.003

51. Obirikorang Y, Obirikorang C, Acheampong E, et al. Adherence to lifestyle modification among hypertensive clients: a descriptive cross-sectional study. Open Access Library J. 2018;5(2):1-13.

52. Okonta HI, Ikombele JB, Ogunbanjo GA. Knowledge, attitude and practice regarding lifestyle modification in type 2 diabetic patients. African j Primary Health Care Family Med. 2014;6 (1):1-6. doi:10.4102/phcfm.v6i1.655

53. Oparah SK, Uhegbu VM, Etokidem AJ. Knowledge of stroke among at risk persons: a survey of patients at a tertiary hospital in Calabar, Southern Nigeria.

54. Poongothai. R. Risk awareness and knowledge of stroke among hypertensive patients. Int J Sci Res. 2017;6(7):2.

55. Rajan J. Knowledge, attitude and practice of hypertension among hypertensive patients in a tertiary care teaching hospital. Int J Basic Clin Pharmacol. 2019;8(5):5.

56. Rajasekharan D, Kulkarni V, Unnikrishnan B, Kumar N, Holla R, Thapar R. Self care activities among patients with diabetes attending a tertiary care hospital in Mangalore Karnataka, India. Ann Med Health Sci Res. 2015;5(1):59-64. doi:10.4103/21419248.149791

57. Rashidi Y, Manaflouyan H, Azar FP, Nikniaz Z, Nikniaz L, Ghaffari S. Knowledge, attitude and practice of Iranian hypertensive patients regarding hypertension. J Cardiovascular Thoracic Res. 2018;10(1):14. doi:10.15171/jcvtr.2018.02

58. Selcuk Ozkan NA. Stroke awareness in people with hypertension. Medicine. 2019;8(3):651-654.

59. Sheeba S, Sneha A, Veena B. Knowledge and self-care practices among diabetics. Asian J Pharm Clin Res. 2017;10(12):234-237. doi:10.22159/ajpcr.2017.v10i12.21072

60. Tesema S, Disasa B, Kebamo S, Kadi E. Knowledge, attitude and practice regarding lifestyle modification of hypertensive patients at Jimma University specialized hospital, Ethiopia. Prim Health Care. 2016;6(1):218-221.

61. Venkatesh U, Srivastava D. Knowledge, attitude and practice in relation to stroke: comparative study between hypertensive and non-hypertensive patients attending a tertiary care centre in Gorakhpur, Uttar Pradesh. India J Med Sci Clin Res. 2016;4:15014-15021. doi:10.18535/jmscr/v4i12.116

62. Vincent-Onabajo G, Mshelia JY, Abubakar U, et al. Knowledge of stroke risk factors among individuals diagnosed with hypertension and diabetes: a hospital-based survey. J Adv Med Med Res. 2015;3:1-8
63. Wahab KW, Kayode OO, Musa OI. Knowledge of stroke risk factors among Nigerians at high risk. J Stroke Cerebrovascular Dis. 2015;24(1):125-129. doi:10.1016/j.jstrokecerebrovasdis.20 14.07 .053

64. Warren-Findlow J, Seymour RB. Prevalence rates of hypertension self-care activities among African Americans. J Natl Med Assoc. 2011;103(6):503-512. doi:10.1016/S0027-9684(15)30365-5

65. Weltermann BM, Driouach-Bleckmann Y, Reinders S, Berndt P, Gesenhues S. Stroke knowledge among diabetics: a cross-sectional study on the influence of age, gender, education, and migration status. BMC Neurol. 2013;13(1):202 . doi:10.1186/ 1471-2377-13-202

66. Bollampally M, Chandershekhar P, Kumar K, Surakasula A, Srikanth S, Reddy T. Assessment of patient's knowledge, attitude and practice regarding hypertension. Int J Res Med Sci. 2016;4 (6):3299-3304. doi:10.18203/2320-6012.ijrms20162283

67. Metias MM, Eisenberg N, Clemente MD, et al. Public health campaigns and their effect on stroke knowledge in a high-risk urban population: a five-year study. Vascular. 2017;25 (5):497-503. doi:10.1177/1708538117691879

68. Mutyambizi C, Pavlova M, Hongoro C, Groot W. Inequalities and factors associated with adherence to diabetes self-care practices amongst patients at two public hospitals in Gauteng, South Africa. BMC Endocr Disord. 2020;20(1):15. doi:10.1186/ s12902-020-0492-y

69. Chimberengwa PT, Naidoo M. Knowledge, attitudes and practices related to hypertension among residents of a disadvantaged rural community in southern Zimbabwe. PLoS One. 2019;14(6): e0215500. doi:10.1371/journal.pone.0215500

70. Kalkonde YV, Alladi S, Kaul S, Hachinski V. Stroke prevention strategies in the developing world. Stroke. 2018;49 (12):3092-3097. doi:10.1161/STROKEAHA.118.017384

71. Wang J, Wen X, Li W, Li X, Wang Y, Lu W. Risk factors for stroke in the Chinese population: a systematic review and meta-analysis. J Stroke Cerebrovascular Dis. 2017;26 (3):509-517. doi:10.1016/j.jstrokecerebrovasdis.2016.12.002

72. Harriott AM, Karakaya F, Ayata C. Headache after ischemic stroke: a systematic review and meta-analysis. Neurology. 2020;94(1):e75-e86. doi:10.1212/WNL.0000000000008591

73. Rathore SS, Hinn AR, Cooper LS, Tyroler HA, Rosamond WD. Characterization of incident stroke signs and symptoms: findings from the atherosclerosis risk in communities study. Stroke. 2002;33 (11):2718-2721. doi:10.1161/01.STR.0000035286.87503.31

74. American stroke association. Stroke Symptoms; 2020. Available from: https://www.stroke.org/en/about-stroke/stroke-symptoms. Accessed June 7, 2020.

75. Nepal G, Yadav JK, Basnet B, Shrestha TM, Kharel G, Ojha R. Status of prehospital delay and intravenous thrombolysis in the management of acute ischemic stroke in Nepal. BMC Neurol. 2019;19(1):155. doi:10.1186/s12883-019-1378-3

76. Kim YS, Park -S-S, Bae H-J, et al. Stroke awareness decreases prehospital delay after acute ischemic stroke in Korea. $B M C$ Neurol. 2011;11(1):2. doi:10.1186/1471-2377-11-2

77. Bray J, Finn J, Cameron P, et al. Stroke public awareness campaign are associated with improved ambulance use for stroke and tia in Victoria, Australia. Circulation. 2017;136(suppl_1):A16 355-A16355.

78. Vo LP, Souksavong JH, Tran A, Chang J, Lor KB. Impact of the Act FAST stroke campaign delivered by student pharmacists on the primary prevention of stroke. J Am Pharmacists Assoc. 2017;57(3):326-332. doi:10.1016/j.japh.2017.02.026

79. Nordanstig A, Asplund K, Norrving B, Wahlgren N, Wester P, Rosengren L. Impact of the Swedish National Stroke Campaign on stroke awareness. Acta Neurol Scand. 2017;136(4):345-351. doi:10.1111/ane.12777 
80. Hartigan I, O’Connell E, O’Brien S, et al. The Irish national stroke awareness campaign: a stroke of success? Applied Nursing Res. 2014;27(4):e13-e19. doi:10.1016/j.apnr.2014.05.004

81. Advani R, Naess H, Kurz M. Mass media intervention in western Norway aimed at improving public recognition of stroke, emergency response, and acute treatment. J Stroke Cerebrovasc Dis. 2016;25 (6):1467-1472. doi:10.1016/j.jstrokecerebrovasdis.2016.02.026

82. Sarfo FS, Mobula LM, Plange-Rhule J, Ansong D, Ofori-Adjei D. Incident stroke among Ghanaians with hypertension and diabetes: a multicenter, prospective cohort study. $J$ Neurol Sci. 2018;395:17-24. doi:10.1016/j.jns.2018.09.018

83. Kumar D. Association of modifiable risk factors with ischemic stroke among hypertensive patients (case control study at tertiary care hospital, Karachi). Int J Nur \& Hos Car. 2018;1:3-07.

84. Huangfu X, Zhu Z, Zhong C, et al. Smoking, hypertension, and their combined effect on ischemic stroke incidence: a prospective study among inner Mongolians in China. J Stroke Cerebrovascular Dis. 2017;26(12):2749-2754. doi:10.1016/j. jstrokecerebrovasdis.2017.06.048

85. Nakamura K, Nakagawa H, Sakurai M, et al. Influence of smoking combined with another risk factor on the risk of mortality from coronary heart disease and stroke: pooled analysis of 10 Japanese cohort studies. Cerebrovascular Dis. 2012;33 (5):480-491. doi:10.1159/000336764

86. Sundseth A, Faiz KW, Rønning OM, Thommessen B. Factors related to knowledge of stroke symptoms and risk factors in a Norwegian stroke population. J Stroke Cerebrovascular Dis. 2014;23 (7):1849-1855. doi:10.1016/j.jstrokecerebrovasdis.2014.02.026

87. Ramírez-Moreno J, Vega PM, Espada S, Alberca SB, Aguirre J, Peral D. Association between self-perceived psychological stress and transitory ischaemic attack and minor stroke: a case-control study. Neurología. 2019;2:798.

88. Gradus JL, Farkas DK, Svensson E, et al. Associations between stress disorders and cardiovascular disease events in the Danish population. BMJ Open. 2015;5(12):e009334. doi:10.1136/bmjopen-2015-009334

89. Kotlęga D, Gołąb-Janowska M, Masztalewicz M, Ciećwież S, Nowacki P. The emotional stress and risk of ischemic stroke. Neurol Neurochir Pol. 2016;50(4):265-270. doi:10.1016/j. pjnns.2016.03.006

90. Jood K, Redfors P, Rosengren A, Blomstrand C, Jern C. Selfperceived psychological stress and ischemic stroke: a case-control study. BMC Med. 2009;7(1):53. doi:10.1186/1741-7015-7-53

91. Pan B, Jin X, Jun L, Qiu S, Zheng Q, Pan M. The relationship between smoking and stroke: a meta-analysis. Medicine. 2019;98:12. doi:10.1097/MD.0000000000014872

92. Monica W, Widyaningsih V, Sulaeman ES. Meta-analysis on the effects of hypertension, type ii diabetes mellitus, and smoking on the risk of stroke among asian young adult Population. J Epidemiol Public Health. 2019;4(4):296-306. doi:10.26911/ jepublichealth.2019.04.04.04
93. Prior PL, Suskin N. Exercise for stroke prevention. Stroke Vascular Neurol. 2018;3(2):59-68. doi:10.1136/svn-2018-000155

94. Middleton LE, Corbett D, Brooks D, et al. Physical activity in the prevention of ischemic stroke and improvement of outcomes: a narrative review. Neurosci Biobehav Rev. 2013;37(2):133-137. doi:10.1016/j.neubiorev.2012.11.011

95. Autenrieth CS, Evenson KR, Yatsuya H, Shahar E, Baggett C, Rosamond WD. Association between physical activity and risk of stroke subtypes: the atherosclerosis risk in communities study. Neuroepidemiology. 2013;40(2):109-116. doi:10.1159/000342151

96. García Ruiz R, Silva Fernández J, García Ruiz RM, et al. Response to symptoms and prehospital delay in stroke patients. Is it time to reconsider stroke awareness campaigns? J Stroke Cerebrovasc Dis. 2018;27(3):625-632. doi:10.1016/j. jstrokecerebrovasdis.2017.09.036

97. Arnett DK, Blumenthal RS, Albert MA, et al. 2019 ACC/AHA guideline on the primary prevention of cardiovascular disease: a report of the American College of Cardiology/American Heart Association Task Force on Clinical Practice Guidelines. $J$ Am Coll Cardiol. 2019;74(10):e177-e232.

98. Cho J-H, Rhee E-J, Park SE, et al. Maintenance of body weight is an important determinant for the risk of ischemic stroke: a nationwide population-based cohort study. PLoS One. 2019;14 (1):e0210153. doi:10.1371/journal.pone.0210153

99. Larsson SC, Wallin A, Wolk A, Markus HS. Differing association of alcohol consumption with different stroke types: a systematic review and meta-analysis. BMC Med. 2016;14(1):178. doi:10. 1186/s12916-016-0721-4

100. Christensen AI, Nordestgaard BG, Tolstrup JS. Alcohol intake and risk of ischemic and haemorrhagic stroke: results from a Mendelian randomisation study. J Stroke. 2018;20(2):218. doi: $10.5853 /$ jos. 2017.01466

101. Cunningham SA, Mosher A, Judd SE, et al. Alcohol consumption and incident stroke among older adults. J Gerontol. 2018;73 (4):636-648. doi:10.1093/geronb/gbw153

102. Pandian JD, Gall SL, Kate MP, et al. Prevention of stroke: a global perspective. Lancet. 2018;392(10154):1269-1278. doi:10.1016/S0140-6736(18)31269-8

103. Niewada M, Michel P. Lifestyle modification for stroke prevention: facts and fiction. Curr Opin Neurol. 2016;29(1):9-13. doi:10.1097/WCO.0000000000000285

104. Spence JD. Nutrition in stroke prevention. Paper presented at: seminars in neurology; 2017

105. Tsivgoulis G, Psaltopoulou T, Wadley VG, et al. Adherence to a Mediterranean diet and prediction of incident stroke. Stroke. 2015;46(3):780-785. doi:10.1161/STROKEAHA.114.007894

\section{Publish your work in this journal}

Risk Management and Healthcare Policy is an international, peerreviewed, open access journal focusing on all aspects of public health, policy, and preventative measures to promote good health and improve morbidity and mortality in the population. The journal welcomes submitted papers covering original research, basic science, clinical \& epidemiological studies, reviews and evaluations, guidelines, expert opinion and commentary, case reports and extended reports. The manuscript management system is completely online and includes a very quick and fair peer-review system, which is all easy to use. Visit http://www.dovepress.com/testimonials.php to read real quotes from published authors. 